\title{
Localized Extramedullary Plasmacytoma: A Report of Two Cases
}

\author{
Shams M Al Otaibi ${ }^{\mathrm{a}, \mathrm{d}}$, Abdul Latif Khan ${ }^{\mathrm{b}}$, Mohammed S Halawanic, \\ Malak A Al Saifa, Mohammed J Al Mahdic
}

\begin{abstract}
Extramedullary plasmacytoma (EMP) is a rare form of plasma cell neoplasm that originates from B lymphocytes. It accounts for only $4 \%$ of all plasma cell disorders. We present two cases of EMP that were diagnosed in two different locations. The first case is a 55-yearold presented with epistaxis and sinusoidal polyps. Histologic examination disclosed plasmacytoma. The second case is a 69-year-old patient presented with upper airway obstruction caused by laryngeal mass and histologic examination revealed plasmacytoma of the larynx. Our cases are unique in their presentation and anatomic locations. To our knowledge, sinusoidal and laryngeal occurrences of the disease are only reported in a handful of cases. Both cases were treated mainly with radiotherapy. The postoperative phase was uneventful.
\end{abstract}

Keywords: Extramedullary plasmacytoma; Plasma cell dyscrasias

\section{Introduction}

Plasma cell dyscrasias (PCD) is the process of abnormal accumulation of monoclonal plasma cells. These cells usually produce high levels of monoclonal immunoglobulins and paraproteins [1]. The PCD encompass a wide spectrum of clinical entities ranging from monoclonal gammopathy of undetermined significance (MGUS) to symptomatic multiple myeloma (MM). Solitary plasmacytoma is the localized neoplastic monoclonal plasma cells accumulation in the absence of systemic plasma cell proliferative disease symptoms such as

Manuscript submitted September 27, 2018, accepted October 8, 2018

${ }^{a}$ College of Medicine at King Saud bin Abdul-Aziz University for Health Sciences, National Guard Health Affairs, Riyadh, Saudi Arabia

bDepartment of Anatomic Pathology, King Abdul-Aziz Medical City, Riyadh, Saudi Arabia

'Department of Otolaryngology- Head and Neck Surgery, King Abdul-Aziz Medical City, Riyadh, Saudi Arabia

${ }^{\mathrm{d} C o r r e s p o n d i n g ~ A u t h o r: ~ S h a m s ~ M ~ A l ~ O t a i b i, ~ C o l l e g e ~ o f ~ M e d i c i n e ~ a t ~ K i n g ~}$ Saud bin Abdul-Aziz University for Health Sciences, National Guard Health Affairs, Riyadh, Saudi Arabia. Email: shams1994@windowslive.com

doi: https://doi.org/10.14740/jmc3179 anemia, hypercalcemia, and renal insufficiency [1]. According to the literature [2], solitary plasmacytoma is a rare form of plasma cell neoplasm and it comprises less than $5 \%$ of all PCD. Solitary plasmacytoma is classified into two groups based on the site of involvement; solitary plasmacytoma of the bone (SPB) and extramedullary plasmacytoma (EMP). SPB occurs when the disease involves osseous tissue, more commonly the axial skeleton, while EMP develops when the disease involves extra-osseous tissue, more commonly the head and neck area [1]. The solitary plasmacytoma exhibits different natural behaviors based on the type. EMP is usually localized and can be managed with radiation therapy. On the other hand, SBP tends to readily convert to multiple myeloma [3].

We hereby report two cases of plasmacytoma in two peculiar locations; the first patient had plasmacytoma in the sinus polyps and the second case had plasmacytoma in the larynx. We discuss the presentation, diagnosis and management of each case. We state the rarity of the disease and the literature review.

\section{Case Reports}

\section{Case 1}

A 55-year-old male presented with epistaxis and nasal crusting. He had a history of chronic sinusitis with right nasal polyp for 1-year duration. He underwent nasal surgery in another center. Then he visited our department (Fig. 1), endoscopic sinus surgery for biopsy and excision of the polyp were carried out and specimens were sent for laboratory. Microscopic examination showed a diffuse monotonous and atypical plasma cell proliferation which manifested the following findings; nuclear hyperchromasia, distinct nucleoli and scattered mitoses. On immunohistochemistry (Fig. 2), these cells were positive for CD38, CD138 and CD79a, and showed kappa light chain restriction. Occasional cells were CD56 positive. The cells were negative for CD45, CD20, EMA, CD3, and lambda. Based on clinicopathological examination, the diagnosis of plasmacytoma was made. The patient was negative for multiple myeloma upon further workup. The patient was asymptomatic in the subsequent clinical visits. After 2 months, the patient developed a right supraclavicular mass. A fine needle aspiration was performed. Cytological examination confirmed the diagnosis of plasmacytoma. The tumor board of lymphoma 


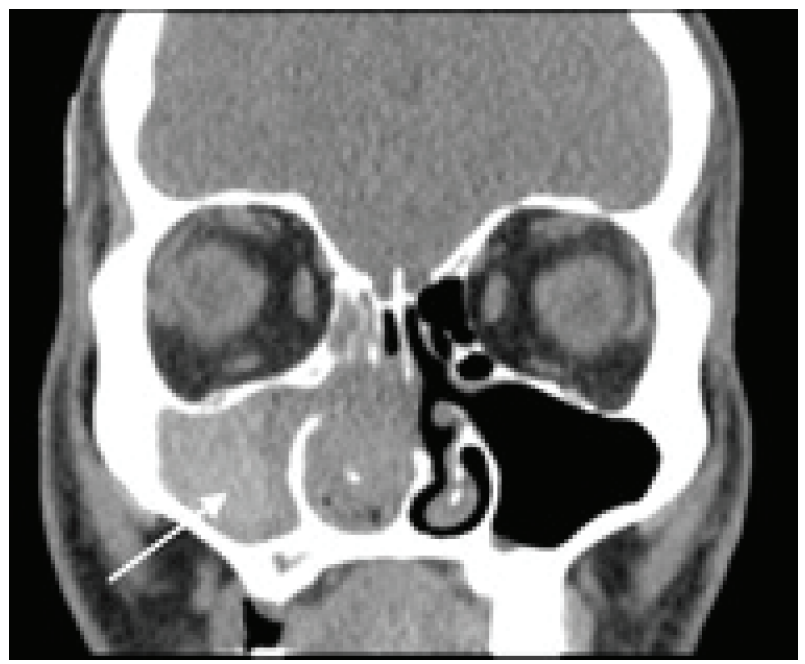

Figure 1. CT showing right nasal sinus opacification (see arrow).

agreed on curative radiation therapy for the patient. The patient is currently followed up with radiation oncology department for radiotherapy. The patient is on $40 \mathrm{~Gy}$ in 20 fractions for the right maxillary tumor and $30 \mathrm{~Gy}$ in 15 fractions for right neck lymph node.

\section{Case 2}

A 69-year-old male with a past history of isolated plasmacytoma of the thyroid presented to the emergency department with difficulty in breathing. Physical examination showed upper airway obstruction by a laryngeal mass (Fig. 3). Awake fiberoptic intubation under sedation was performed. The operation took place and biopsy of laryngeal mass was sent for histopathology. Microscopic examination of laryngeal mass cells showed neoplastic proliferation of round blue cells with a plasmacytic morphology. On immunohistochemistry (Fig. 4), these cells were positive for CD45, CD38, CD138, CD79a and kappa. The cells were negative for CD20, CD3, CD56, and lambda. Based on clinicopathological findings, the diagnosis of plasmacytoma was made. The possibility of systemic disease (multiple myeloma) was excluded by additional workup.

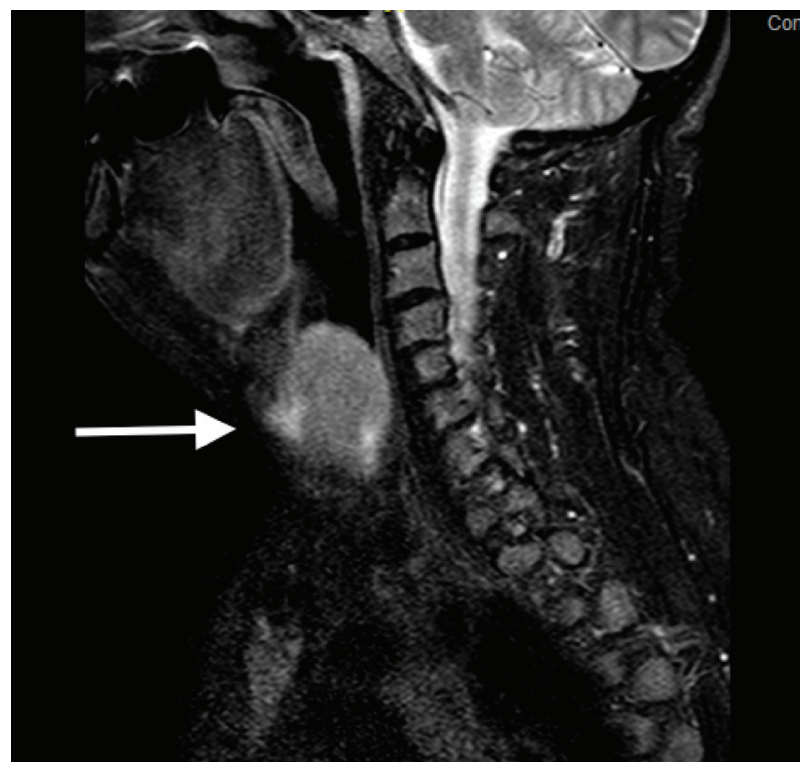

Figure 3. MRI showing laryngeal mass obstructing airway (see arrow).

The patient was treated with localized radiation of 45 Gy in 25 fractions directed to right laryngeal mass. The patient was asymptomatic in the successive follow-up visits of 6 months.

\section{Discussion}

EMP is a rare form of plasma cell neoplasm that is deriving from B lymphocytes [4]. The first EMP report was made in 1905 by Schridde [5]. Isolated EMPs account for only 4\% of all plasma cell disorders [5]. According to Ozturk et al [4], $80 \%$ of EMPs reside in the head and neck area, more commonly the upper respiratory tract and oral cavity. There is $3: 1$ preponderance of male in the incidence, and EMP is diagnosed later in their life, commonly in the fourth to seventh decade [4, $6,7]$. This is consistent with our cases, as both cases were diagnosed in their fifth and sixth decade. The exact etiology of the disease is not fully understood yet; however, several contributing factors have been indicated, such as viral pathogenesis and chronic irritation from various inhaled substances [6].
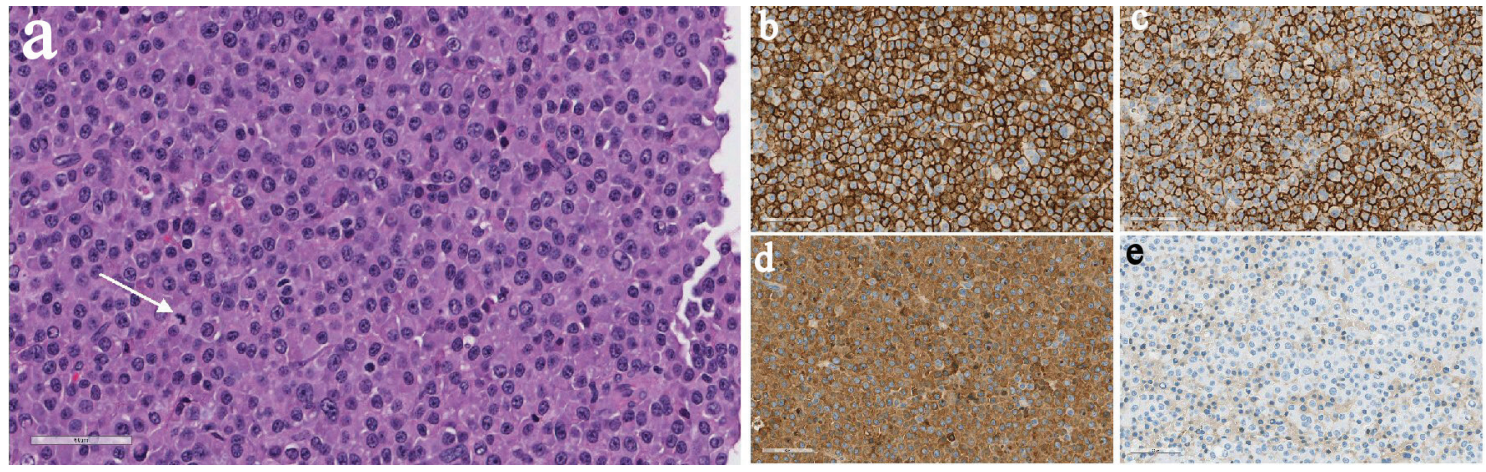

Figure 2. (a) H\&E slide $(\times 400)$ showing diffused monotonous plasmacytoid cells with mitosis (see arrow). On immunohistochemistry ( $\times 400)$, the cells are positive for CD38 (b), CD138 (c), and kappa (d). The cells are negative for lambda (e). 

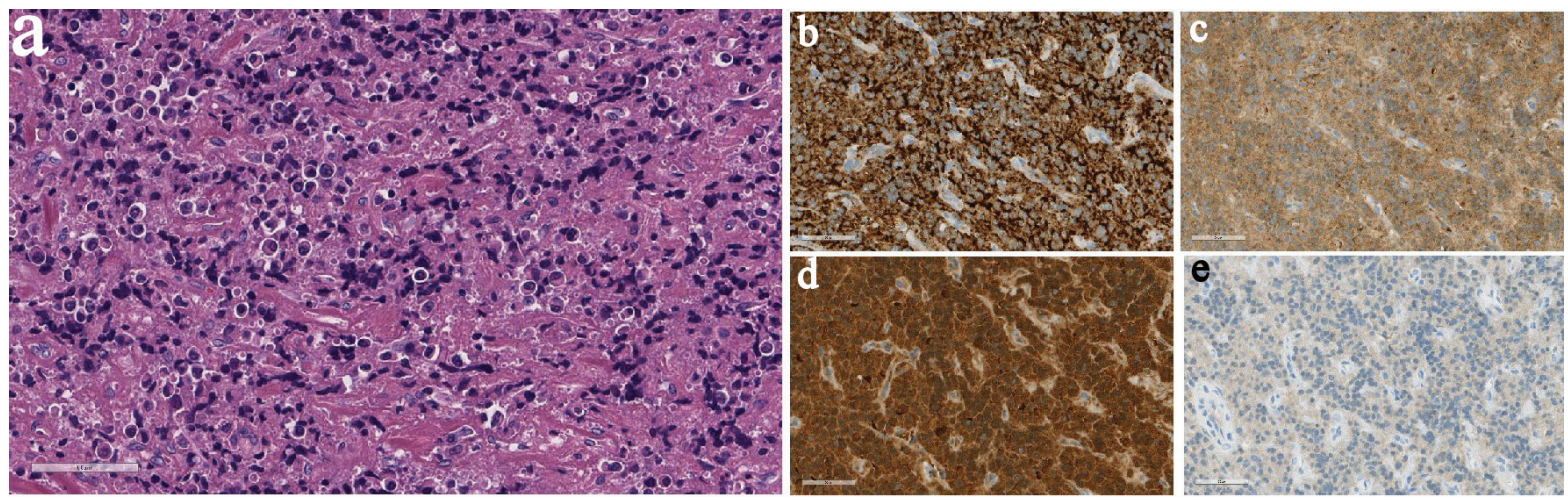

Figure 4. (a) H\&E slide $(\times 400)$ showing cauterized tissue with diffuse infiltrate of neoplastic plasma cells. On immunohistochemistry ( $\times 400)$, the cells are positive for CD38 (b), CD138 (c), and kappa (d). The cells are negative for lambda (e).

The clinical presentation of EMP is dependent on the location of soft tissue mass. Eight percent of EMPs involve mucosa-associated lymphoid tissue (MALT) of upper respiratory tract; $75 \%$ of the MALT is located in the oro-nasopharynx and paranasal sinuses. Therefore, most of the patients present with nasal obstruction, rhinorrhea and epistaxis [1]. Ozturk et al reported the frequent sites of the disease as follows; nasopharynx (75\%), larynx (18\%) and oropharynx (12\%) [4]. Upon reviewing Chinese literature [8], case series of 32 cases showed the most common sites: upper aerodigestive tract, accounted for 21 cases $(65.6 \%)$; six cases for skin $(18.8 \%)$, and two cases for tonsil $(6.3 \%)$. Other rare sites include: gastrointestinal tract, ovary and lung [1]. In 1999, literature review of 714 cases of EMP by Alexiou et al delineated that paranasal sinus accounted for 154 cases $(21.5 \%)$ and only 43 cases in the larynx (6\%) [5]. Based on the literature, our cases manifested the plasmacytoma in unusual sites like larynx and nasal sinusoidal polyps. The diagnosis of EMP is made if the following criteria are met: 1) A plasma cell tumor biopsy that involves a single extramedullary site with or without lymph node involvement; 2) A biopsy of bone marrow that indicates less than $5 \%$ of plasma cells; 3) No additional lesions on the skeletal survey; 4) Absence of Bence-Jones protein in the urine [5]. Thorough diagnostic procedures such as computed tomography (CT), magnetic resonance imaging (MRI) and endoscopies are needed to identify the extent of the disease [5]. We follow the same criteria for our patients, as we did bone marrow biopsy to exclude multiple myeloma. Moreover, Bence-Jones protein and radiological workups came out as negative results. In addition to the morphologic histopathology, immunohistochemistry is essential for diagnosis of EMP due to the difficulty in distinguishing EMP from the other differential diagnoses [5].

Radical surgery is recommended to be avoided in head and neck EMP, as radiotherapy is the main treatment. EMP tumors are highly radio-sensitive with longstanding local control of 79 to $91 \%$ after radiation therapy [9]. Based on the International Lymphoma Radiation Oncology Group (ILROG) [9], there is still no well-developed standardized radiation dosage. However, 40 to $45 \mathrm{~Gy}$ is the common dosage used in clinical practice [9]. Studies have shown that local control rate with 40 Gy and above was 94\%; whereas with dosages less than $40 \mathrm{~Gy}$, the local control rate lowered to $69 \%$ [9]. Therefore, the current guidelines for radiation therapy of EMP suggest the dosage of 40 to $50 \mathrm{~Gy}$. This is in alignment with our cases, in which dosage of 40 Gy was used in both cases. Nodal involvement occurs in $25 \%$ of head and neck EMP [9]. Radiation therapy remains the optimal treatment for the involved nodes. The implement of prophylactic radiation therapy for potential nodes is still debatable [9]. Historically, radiation coverage of the regional nodes was routinely performed. However, with the advanced radiological tool nowadays, ILROG recommends the usage of elective radiation therapy to be limited to high-risk cases [9].

\section{Conflict of Interest}

The authors declare that there is no conflict of interest.

\section{References}

1. Tandon N, Kumar SK. Plasmacytoma current approach to diagnosis and management $\mathrm{S}$. In: Zimmerman TM, Kumar SK. Biology and management of unusual plasma cell dyscrasias. New York: Springer; 2017. p. 17-39.

2. Dimopoulos MA, Moulopoulos A, Delasalle K, Alexanian R. Solitary plasmacytoma of bone and asymptomatic multiple myeloma. Hematol Oncol Clin North Am. 1992;6(2):359-369.

3. Holland J, Trenkner DA, Wasserman TH, Fineberg B. Plasmacytoma. Treatment results and conversion to myeloma. Cancer. 1992;69(6):1513-1517.

4. Ozturk K, Oahin M, Midilli R, Gursan G, Ozsan N, and Savao R. Extramedullary plasmacytoma of head and neck region: report of six cases with different localizations. Otorhinolaryngology Clinics - An International Journal. 2013;5:157-163.

5. Alexiou C, Kau RJ, Dietzfelbinger H, Kremer M, Spiess JC, Schratzenstaller B, Arnold W. Extramedullary plasmacytoma: tumor occurrence and therapeutic concepts. Cancer. 1999;85(11):2305-2314.

6. Chang YL, Chen PY, Hung SH. Extramedullary plasmacytoma of the nasopharynx: A case report and review of 
the literature. Oncol Lett. 2014;7(2):458-460.

7. Woodruff RK, Whittle JM, Malpas JS. Solitary plasmacytoma. I: Extramedullary soft tissue plasmacytoma. Cancer. 1979;43(6):2340-2343.

8. Zuo Z, Tang Y, Bi CF, Zhang WY, Zhao S, Wang XQ, Yang QP, et al. Extraosseous (extramedullary) plasmacytomas: a clinicopathologic and immunophenotypic study of 32 Chinese cases. Diagn Pathol. 2011;6:123.

9. Tsang RW, Campbell BA, Goda JS, Kelsey CR, Kirova YM, Parikh RR, Ng AK, et al. Radiation therapy for solitary plasmacytoma and multiple myeloma: guidelines from the International Lymphoma Radiation Oncology Group. Int J Radiat Oncol Biol Phys. 2018;101(4):794808. 